\title{
Local Fractional Fourier Series Method for Solving Nonlinear Equations with Local Fractional Operators
}

\author{
Yong-Ju Yang and Shun-Qin Wang \\ School of Mathematics and Statistics, Nanyang Normal University, Nanyang 473061, China \\ Correspondence should be addressed to Yong-Ju Yang; tomjohn1007@126.com
}

Received 24 October 2014; Accepted 23 January 2015

Academic Editor: Sandile Motsa

Copyright (C) 2015 Y.-J. Yang and S.-Q. Wang. This is an open access article distributed under the Creative Commons Attribution License, which permits unrestricted use, distribution, and reproduction in any medium, provided the original work is properly cited.

We apply the local fractional Fourier series method for solving nonlinear equation with local fractional operators. This method is the coupling of the local fractional Fourier series expansion method with other methods, such as the Yang-Laplace transformation method and the local fractional power series method, which effectively separates the variables of partial differential equation. Some testing nonlinear equations and equation systems are given to demonstrate the accuracy and applicability of the proposed approach.

\section{Introduction}

There are many definitions of fractional derivative and integral, such as Riesz, Caputo, Riemann-Liouville, Marchaud, and Sonin-Letnikov $[1,2]$. Nonlinear fractional differential equation and nonlinear fractional integral-differential equation are the promising fields of research in technology and science. But it is very difficult to solve these nonlinear equations with fractional differential or fractional integral operator. For these reasons, many methods have been developed to solve these nonlinear equations, for example, the homotopy perturbation method $[3,4]$, the differential transform method $[5,6]$, the Adomian decomposition method [7], the shifted Legendre spectral method [8], the variational iteration method [9-11], and the shifted Jacobi-Gauss-Lobatto collocation method [12].

Recently, the local fractional differential and calculus theory is introduced in $[13,14]$, which is set up on fractal geometry and which is the best candidate for depicting the nondifferential function defined on Cantor sets. The geometric and physical interpretation for the local fractional derivative can be seen in [13-16]. The theory in $[13,14]$ has been successfully applied in describing many physical phenomena in fractal-like media; for example, the local fractional Poisson and Laplace equations with applications to electrostatics in fractal domain were expressed in [17]; to describe the fractal electric and magnetic fields, Maxwell's equations on Cantor sets were utilized in [18]; diffusion and wave equations on Cantor sets were investigated in [19]; 1D heat conduction in a fractal medium was discussed in [20]. The local fractional differential and calculus theory has also been applied in some branches of applied mathematics [21], and so forth. Meanwhile, a substantial amount of methods for the fractional differential equations with local fractional operators is proposed, which are referred to in [17-21].

The local fractional Fourier series method has been proposed in [22], which is the coupling of the local fractional Fourier series expansion method with the Yang-Laplace transformation method for solving local fractional linear differential equations. The method has provoked some attention by a few authors in $[23,24]$. In this paper, by coupling of the local fractional Fourier series expansion method with a few methods, not only the Yang-Laplace method, we generalize and enrich the local fractional Fourier series method for solving some nonlinear equations within the local fractional differential or fractional integral operator. In this paper, the advantage of this method can be attributed to its endeavor in finding the solution by transforming solving partial differential or integral-differential equations to solving a system of ordinary differential equations and then reducing the complicated calculations to more easily calculations. 
The rest of the paper is organized as follows. In Section 2, the basic mathematical fundamentals are presented briefly. In Section 3, the local fractional Fourier series method for solving the nonlinear differential equations with local fractional operator is presented. In Section 4, several test examples are illustrated. Finally, in Section 5, the conclusion is given.

\section{Mathematical Fundamentals}

In this section, we introduce the basic definitions and properties of the local fractional differential and calculus theory which will be used in this paper.

2.1. Local Fractional Derivative and Integral. The local fractional derivative of $f(x)$ of order $\alpha(0<\alpha \leq 1)$ at $x_{0}$ is defined by $[13,14]$

$$
\begin{aligned}
f^{(\alpha)}\left(x_{0}\right) & =D_{x}{ }^{(\alpha)} f\left(x_{0}\right)=\left.\frac{d^{\alpha} f(x)}{d x^{\alpha}}\right|_{x=x_{0}} \\
& =\lim _{x \rightarrow x_{0}} \frac{\Gamma(1+\alpha)\left(f(x)-f\left(x_{0}\right)\right)}{\left(x-x_{0}\right)^{\alpha}} .
\end{aligned}
$$

In the bounded closed interval $[a, b]$, the local fractional integral of $f(x)$ of order $\alpha$ is defined by $[13,14]$

$$
\begin{aligned}
a_{a}{ }^{(\alpha)} f(x) & =\frac{1}{\Gamma(1+\alpha)} \lim _{\Delta t \rightarrow 0} \sum_{j=0}^{j=N-1} f\left(t_{j}\right)\left(\Delta t_{j}\right)^{\alpha} \\
& =\frac{1}{\Gamma(1+\alpha)} \int_{a}^{b} f(t)(d t)^{\alpha},
\end{aligned}
$$

where $\Delta t_{j}=t_{j+1}-t_{j}, \Delta t=\max \left\{\Delta t_{1}, \Delta t_{2}, \Delta t_{j}, \ldots\right\}$, and $\left[t_{j}\right.$, $\left.t_{j+1}\right], j=0, \ldots, N-1, t_{0}=a, t_{N}=b$, is a partition of the interval $[a, b]$.

2.2. Basic Concept of Yang-Laplace Transform. Suppose that $(1 / \Gamma(1+\alpha)) \int_{0}^{\infty}|f(x)|(d x)^{\alpha}$ is bounded; the Yang-Laplace transform of $f(x)$ is defined as [14]

$$
\begin{aligned}
& L_{\alpha}\{f(x)\}= f_{s}^{L, \alpha}(s) \\
&=\frac{1}{\Gamma(1+\alpha)} \int_{0}^{\infty} E_{\alpha}\left(-s^{\alpha} x^{\alpha}\right) f(x)(d x)^{\alpha}, \\
& \quad 0<\alpha \leq 1,
\end{aligned}
$$

where the latter integral converges and $s^{\alpha} \in R^{\alpha}$.

The inverse Yang-Laplace transform of $f(x)$ is defined by the expression $[13,14]$ :

$$
\begin{array}{r}
L_{\alpha}^{-1}\left\{f_{s}^{L, \alpha}(s)\right\}=f(t)=\frac{1}{(2 \pi)^{\alpha}} \int_{\beta-i \infty}^{\beta+i \infty} E_{\alpha}\left(s^{\alpha} t^{\alpha}\right) f_{s}^{L, \alpha}(s)(d s)^{\alpha}, \\
0<\alpha \leq 1,
\end{array}
$$

where $s^{\alpha}=\beta^{\alpha}+i^{\alpha} \omega^{\alpha}, i^{\alpha}$ is an imaginary unit and $\operatorname{Re}(s)=\beta>$ 0 .
2.3. Local Fractional Taylor's Series. If $u^{((k+1) \alpha)}(t)$ are local fractional continuous on the interval $(a, b)$ for $k=0,1, \ldots$, $n, \ldots$, the local fractional Taylor's series of $u(t)$ is defined as

$$
u(t)=\sum_{k=0}^{\infty} \frac{u^{(k \alpha)}\left(t_{0}\right)}{\Gamma(1+k \alpha)}\left(t-t_{0}\right)^{k \alpha}
$$

with $a<t_{0}<t<b, \forall t \in(a, b)$, where $u^{((k+1) \alpha)}(t)=$ $\underbrace{D_{t}^{(\alpha)} \cdots D_{t}^{(\alpha)}} u(t)[13,14]$.

$k+1$ times

Suppose $y=f\left(x^{\alpha}\right)$ (or $y=f(x, y)$, resp.) is a function defined on fractal set $\Omega^{\alpha}$ (or $\Omega^{\alpha} \times \Omega^{\alpha}$, resp.). For convenience, in this paper, let $y=f(x), x \in \Omega$ (or $y=f(x, y)$, $(x, y) \in \Omega \times \Omega$, resp.) denote $y=f\left(x^{\alpha}\right), x^{\alpha} \in \Omega^{\alpha}$ (or $y=f\left(x^{\alpha}, y^{\alpha}\right),\left(x^{\alpha}, y^{\alpha}\right) \in \Omega^{\alpha} \times \Omega^{\alpha}$, resp. $)$. For more details of the local fractional differential and calculus theory can be seen in $[13,14]$.

\section{Local Fractional Fourier Series Method}

In this section we will present the local fractional Fourier series method to derive particular solution of some nonlinear differential equations.

In order to elucidate the solution procedure of this method, we consider the local fractional differential equation on fractal set

$$
\frac{\partial^{k_{0} \alpha} u(x, t)}{\partial t^{k_{0} \alpha}}+L[u(x, t)]+R[u(x, t)]=g(x, t)
$$

with boundary and initial conditions

$$
\begin{gathered}
u(0, t)=u(l, t)=0, \\
\frac{\partial^{k^{\prime} \alpha} u(x, o)}{\partial t^{k^{\prime} \alpha}}=\varphi_{k^{\prime}}(x), \quad k^{\prime}=0,1, \ldots, k_{0}-1,
\end{gathered}
$$

where $\partial^{k_{0} \alpha} u(x, t) / \partial t^{k_{0} \alpha}$ is the term of the highest order derivative, $L$ is a linear operator, $R$ is a nonlinear operator, $g(x, t)$ is a source term, and $l>0$.

Now we investigate the solution of (6).

Expand $u(x, t), g(x, t)$ to be odd functions of period $(2 l)^{\alpha}$ in terms of the variable $x^{\alpha}$, respectively (in the following, let $u(x, t)$ also denote its expanded odd function of period $(2 l)^{\alpha}$ for simplicity). According to the local fractional Fourier series expansion method, the Fourier trigonometric series of some functions can be represented by

$$
\begin{aligned}
u(x, t)= & \sum_{n=1}^{\infty} u_{n}(t) \sin _{\alpha} n^{\alpha}\left(\frac{\pi x}{l}\right)^{\alpha}, \\
g(x, t)= & \sum_{n=1}^{\infty} g_{n}(t) \sin _{\alpha} n^{\alpha}\left(\frac{\pi x}{l}\right)^{\alpha}, \\
L u(x, t)= & \sum_{n=1}^{\infty} A_{n}(t) \sin _{\alpha} n^{\alpha}\left(\frac{\pi x}{l}\right)^{\alpha} \\
& +\sum_{n=1}^{\infty} B_{n}(t) \cos _{\alpha} n^{\alpha}\left(\frac{\pi x}{l}\right)^{\alpha},
\end{aligned}
$$




$$
\begin{aligned}
R u(x, t)= & \sum_{n=1}^{\infty} C_{n}(t) \sin _{\alpha} n^{\alpha}\left(\frac{\pi x}{l}\right)^{\alpha} \\
& +\sum_{n=1}^{\infty} D_{n}(t) \cos _{\alpha} n^{\alpha}\left(\frac{\pi x}{l}\right)^{\alpha},
\end{aligned}
$$

where the functions coefficients $u_{n}(t), g_{n}(t), A_{n}(t), B_{n}(t)$, $C_{n}(t)$, and $D_{n}(t)$ are determined by the following equation system:

$$
\begin{gathered}
u_{n}(t)=\frac{2^{\alpha}}{l^{\alpha}} \int_{0}^{l} u(x, t) \sin _{\alpha} n^{\alpha}\left(\frac{\pi x}{l}\right)^{\alpha}(d x)^{\alpha}, \\
g_{n}(t)=\frac{2^{\alpha}}{l^{\alpha}} \int_{0}^{l} g(x, t) \sin _{\alpha} n^{\alpha}\left(\frac{\pi x}{l}\right)^{\alpha}(d x)^{\alpha}, \\
A_{n}(t)=\frac{1^{\alpha}}{l^{\alpha}} \int_{-l}^{l} L u(x, t) \sin _{\alpha} n^{\alpha}\left(\frac{\pi x}{l}\right)^{\alpha}(d x)^{\alpha}, \\
B_{n}(t)=\frac{1^{\alpha}}{l^{\alpha}} \int_{-l}^{l} L u(x, t) \cos _{\alpha} n^{\alpha}\left(\frac{\pi x}{l}\right)^{\alpha}(d x)^{\alpha}, \\
C_{n}(t)=\frac{1^{\alpha}}{l^{\alpha}} \int_{-l}^{l} R u(x, t) \sin _{\alpha} n^{\alpha}\left(\frac{\pi x}{l}\right)^{\alpha}(d x)^{\alpha}, \\
D_{n}(t)=\frac{1^{\alpha}}{l^{\alpha}} \int_{-l}^{l} R u(x, t) \cos _{\alpha} n^{\alpha}\left(\frac{\pi x}{l}\right)^{\alpha}(d x)^{\alpha} .
\end{gathered}
$$

Substituting (9) into (6) and assuming that termwise differential is permitted, we obtain

$$
\begin{aligned}
& \sum_{n=1}^{\infty} u_{n}^{\left(k_{0} \alpha\right)}(t) \sin _{\alpha} n^{\alpha}\left(\frac{\pi x}{l}\right)^{\alpha}+\sum_{n=1}^{\infty} A_{n}(t) \sin _{\alpha} n^{\alpha}\left(\frac{\pi x}{l}\right)^{\alpha} \\
& +\sum_{n=1}^{\infty} B_{n}(t) \cos _{\alpha} n^{\alpha}\left(\frac{\pi x}{l}\right)^{\alpha} \\
& +\sum_{n=1}^{\infty} C_{n}(t) \sin _{\alpha} n^{\alpha}\left(\frac{\pi x}{l}\right)^{\alpha} \\
& +\sum_{n=1}^{\infty} D_{n}(t) \cos _{\alpha} n^{\alpha}\left(\frac{\pi x}{l}\right)^{\alpha} \\
& =\sum_{n=1}^{\infty} g_{n}(t) \sin _{\alpha} n^{\alpha}\left(\frac{\pi x}{l}\right)^{\alpha} .
\end{aligned}
$$

Comparing the coefficient of like $\sin _{\alpha} n^{\alpha} x^{\alpha}$ and $\cos _{\alpha} n^{\alpha} x^{\alpha}$ on both sides of (10), respectively, the following equation system is obtained:

$$
\begin{gathered}
u_{n}^{\left(k_{0} \alpha\right)}(t)+A_{n}(t)+C_{n}(t)=g_{n}(t), \\
B_{n}(t)+D_{n}(t)=0 .
\end{gathered}
$$

Indeed, via the local fractional Fourier series expansion, the partial differential equation (6) problem is attributed to the ordinary equation system (11). Obviously, it is easier to study system (11) than (6). Some other methods, for example, the Yang-Laplace transformation method and the local fractional power series method, can be easily selected to solve (11).

\section{Illustrative Examples}

In order to illustrate the above local fractional Fourier series method in Section 3, we give the following several examples on fractal set: the local fractional differential equation; integral-differential equation; and integral-differential equation system.

Example 1. The nonhomogeneous local fractional differential Tricomi equation is written in the following form:

$$
\frac{\partial^{2 \alpha} u(x, t)}{\partial t^{2 \alpha}}+t^{\alpha} \frac{\partial^{2 \alpha} u(x, t)}{\partial x^{2 \alpha}}=x^{\alpha}
$$

subject to the boundary and initial conditions described by

$$
\begin{gathered}
u(x, 0)=0, \\
\frac{\partial^{\alpha} u(x, o)}{\partial t^{\alpha}}=0, \\
u(0, t)=u(\pi, t)=0 .
\end{gathered}
$$

Obviously

$$
\begin{gathered}
L[u(x, t)]=0 ; \quad R[u(x, t)]=t^{\alpha} \frac{\partial^{2 \alpha} u(x, t)}{\partial x^{2 \alpha}} ; \\
g(x, t)=x^{\alpha} .
\end{gathered}
$$

According to the equation system (8) and (9), we obtain

$$
\begin{gathered}
A_{n}(t)=B_{n}(t)=D_{n}(t)=0 ; \\
u(x, t)=\sum_{n=1}^{\infty} u_{n}(t) \sin _{\alpha} n^{\alpha} x^{\alpha} ; \\
x^{\alpha}=\sum_{n=1}^{\infty}(-1)^{n+1} \frac{2^{\alpha}}{n^{\alpha}} \sin _{\alpha}(n x)^{\alpha} .
\end{gathered}
$$

Substituting (16) and (17) into (12) and then comparing the coefficient of like $\sin _{\alpha} n^{\alpha} x^{\alpha}$, the following equation can be deduced:

$$
u_{n}^{(2 \alpha)}(t)=n^{2 \alpha} t^{\alpha} u_{n}(t)+(-1)^{n+1} \frac{2^{\alpha}}{n^{\alpha}} .
$$

Applying the Yang-Laplace transform on both sides of (18) and using the initial condition (13), we have

$$
L_{\alpha}\left[u_{n}(t)\right]=\frac{n^{2 \alpha}}{s^{2 \alpha}}\left\{L_{\alpha}\left[u_{n}(t)\right]\right\}^{\prime}+(-1)^{n+1} \frac{2^{\alpha}}{n^{\alpha}} \frac{1^{\alpha}}{s^{\alpha}} .
$$

Taking the inverse Yang-Laplace transform on both sides of (19), we get

$$
u_{n}(t)=L_{\alpha}^{-1}\left[E_{\alpha}\left(\int \frac{s^{2 \alpha}}{n^{2 \alpha}}(d s)^{\alpha}\right)_{\alpha} \int(-1)^{n} \frac{2^{\alpha}}{n^{3 \alpha}}(d s)^{\alpha}\right] .
$$

Thus, the final solution of (12) is

$$
\begin{aligned}
u(x, t)=\sum_{n=1}^{\infty} L_{\alpha}^{-1}[ & E_{\alpha}\left(\int \frac{s^{2 \alpha}}{n^{2 \alpha}}(d s)^{\alpha}\right)_{\alpha} \\
& \left.\cdot \int(-1)^{n} \frac{2^{\alpha}}{n^{3 \alpha}}(d s)^{\alpha}\right] \sin _{\alpha}(n x)^{\alpha} .
\end{aligned}
$$


According to the ideas of local fractional Fourier series method, we can also deal with integral-differential equation or integral-differential equation system with a similar method.

Example 2. We consider the following gas dynamic-like integral-differential equation:

$$
\begin{aligned}
& \frac{\partial^{2 \alpha} u(x, t)}{\partial t^{2 \alpha}}-\frac{1^{\alpha}}{(2 h)^{\alpha}} \int_{x-h}^{x+h} u(\mu, t)(d \mu)^{\alpha}-u(x, t) \frac{\partial^{2 \alpha} u(x, t)}{\partial x^{2 \alpha}} \\
& -u(x, t)\left(1^{\alpha}+u(x, t)\right)=0,
\end{aligned}
$$

subject to the boundary and initial conditions

$$
\begin{gathered}
u(t, 0)=u(t, \pi)=0, \\
u(x, o)=\frac{\partial^{\alpha} u(x, o)}{\partial t^{\alpha}}=\sin _{\alpha} x^{\alpha} .
\end{gathered}
$$

Obviously

$$
\begin{gathered}
L[u(x, t)]=-\frac{1^{\alpha}}{(2 h)^{\alpha}} \int_{x-h}^{x+h} u(\mu, t)(d \mu)^{\alpha}-u(x, t) ; \\
R[u(x, t)]=-u(x, t) \frac{\partial^{2 \alpha} u(x, t)}{\partial x^{2 \alpha}}-u^{2}(x, t) .
\end{gathered}
$$

According to (8) and (9) and (22), we obtain

$$
\begin{gathered}
B_{n}(t)=D_{n}(t)=0 ; \\
u_{n}^{\prime \prime}(t)=u_{n}(t)+\frac{\sin _{\alpha}(n h)^{\alpha}}{(n h)^{\alpha}} u_{n}+C_{n}(t),
\end{gathered}
$$

where

$$
C_{n}(t)=-\frac{1^{\alpha}}{\pi^{\alpha}} \int_{-\pi}^{\pi}\left(u \frac{\partial^{2 \alpha} u}{\partial x^{2 \alpha}}+u^{2}\right) \sin _{\alpha} n^{\alpha} x^{\alpha}(d x)^{\alpha} .
$$

By virtue of

$$
u(x, t)=-u(-x, t),
$$

we can get

$$
R u(x, t)=R u(-x, t) .
$$

Due to (28), we have

$$
C_{n}(t)=-\frac{1^{\alpha}}{\pi^{\alpha}} \int_{-\pi}^{\pi}\left(u \frac{\partial^{2 \alpha} u}{\partial x^{2 \alpha}}+u^{2}\right) \sin _{\alpha} n^{\alpha} x^{\alpha}(d x)^{\alpha}=0 .
$$

Then we yield

$$
u_{n}^{(2 \alpha)}(t)=u_{n}(t)+\frac{\sin _{\alpha}(n h)^{\alpha}}{(n h)^{\alpha}} u_{n} .
$$

Analyzing (23) and (30), we impose the following assumptions on (30):

$$
u_{n}(t)=0, \quad n=2,3, \ldots
$$

According to (30), we can get

$$
u_{1}^{(2 \alpha)}(t)=u_{1}(t)+\frac{\sin _{\alpha} h^{\alpha}}{h^{\alpha}} u_{1} .
$$

Applying the eigenvalue method [14], the solution of (32) is

$$
\begin{aligned}
u_{1}(t)= & \lambda_{1} E_{\alpha}\left(\sqrt{1^{\alpha}+\frac{\sin _{\alpha} h^{\alpha}}{h^{\alpha}} t^{\alpha}}\right) \\
& +\lambda_{2} E_{\alpha}\left(-\sqrt{1^{\alpha}+\frac{\sin _{\alpha} h^{\alpha}}{h^{\alpha}}} t^{\alpha}\right),
\end{aligned}
$$

where $\lambda_{1}, \lambda_{2}$ are all constant numbers.

Using the initial condition (23) we get

$$
\begin{aligned}
u_{1}(t)= & \frac{1^{\alpha}}{2^{\alpha}}\left(1^{\alpha}+\frac{1^{\alpha}}{\sqrt{1^{\alpha}+\sin _{\alpha} h^{\alpha} / h^{\alpha}}}\right) \\
& \cdot E_{\alpha}\left(\sqrt{1^{\alpha}+\frac{\sin _{\alpha} h^{\alpha}}{h^{\alpha}} t^{\alpha}}\right) \\
& +\frac{1^{\alpha}}{2^{\alpha}}\left(1^{\alpha}-\frac{1^{\alpha}}{\sqrt{1^{\alpha}+\sin _{\alpha} h^{\alpha} / h^{\alpha}}}\right) \\
& \cdot E_{\alpha}\left(-\sqrt{1^{\alpha}+\frac{\sin _{\alpha} h^{\alpha}}{h^{\alpha}}} t^{\alpha}\right) .
\end{aligned}
$$

By virtue of (31) and (34), the final solution of (22) is readily found to be

$$
\begin{aligned}
u(x, t)=\frac{1^{\alpha}}{2^{\alpha}}\left\{\left(1^{\alpha}+\frac{1^{\alpha}}{\sqrt{1^{\alpha}+\sin _{\alpha} h^{\alpha} / h^{\alpha}}}\right)\right. \\
\cdot E_{\alpha}\left(\sqrt{1^{\alpha}+\frac{\sin _{\alpha} h^{\alpha}}{h^{\alpha}} t^{\alpha}}\right) \\
+\left(1^{\alpha}-\frac{1^{\alpha}}{\sqrt{1^{\alpha}+\sin _{\alpha} h^{\alpha} / h^{\alpha}}}\right) \\
\left.\cdot E_{\alpha}\left(-\sqrt{1^{\alpha}+\frac{\sin _{\alpha} h^{\alpha}}{h^{\alpha}}} t^{\alpha}\right)\right\} \sin _{\alpha} x^{\alpha} .
\end{aligned}
$$

Example 3. The local fractional differential equation system is written in the following form:

$$
\begin{gathered}
\sin _{\alpha} x^{\alpha} \frac{\partial^{\alpha} v(x, t)}{\partial t^{\alpha}}-u(x, t) v(x, t)=\sin ^{2}{ }_{\alpha} x^{\alpha}, \\
\sin _{\alpha} x^{\alpha} \frac{\partial^{\alpha} u(x, t)}{\partial t^{\alpha}}-v^{2}(x, t)=\sin _{\alpha}{ }_{\alpha} x^{\alpha},
\end{gathered}
$$

subject to the boundary and initial conditions described by

$$
\begin{aligned}
& u(x, 0)=v(x, 0)=0, \\
& u(0, t)=u(\pi, t)=0, \\
& v(0, t)=v(\pi, t)=0 .
\end{aligned}
$$


According to (8) and (9), we can get

$$
\begin{aligned}
& u(x, t)=\sum_{n=1}^{\infty} u_{n}(t) \sin _{\alpha} n^{\alpha} x^{\alpha}, \\
& v(x, t)=\sum_{n=1}^{\infty} v_{n}(t) \sin _{\alpha} n^{\alpha} x^{\alpha} .
\end{aligned}
$$

Substituting (38) into (36) results in the following:

$$
\begin{aligned}
& \sin _{\alpha} x^{\alpha} \sum_{n=1}^{\infty} u_{n}^{(\alpha)}(t) \sin _{\alpha} n^{\alpha} x^{\alpha} \\
& =\sum_{n=0}^{\infty} E_{n} \cos _{\alpha} n^{\alpha} x^{\alpha}+\sin _{\alpha}^{2} x^{\alpha}, \\
& \sin _{\alpha} x^{\alpha} \sum_{n=1}^{\infty} v_{n}^{(\alpha)}(t) \sin _{\alpha} n^{\alpha} x^{\alpha} \\
& =\sum_{n=0}^{\infty} F_{n} \cos _{\alpha} n^{\alpha} x^{\alpha}+\sin _{\alpha}^{2} x^{\alpha},
\end{aligned}
$$

where

$$
\begin{gathered}
E_{n}=\frac{1^{\alpha}}{2^{\alpha}} \sum_{m=1}^{\infty} u_{m}\left(v_{m+n}-v_{m-n}\right), \quad n>1, \\
E_{0}=\frac{u_{1} v_{1}}{2^{\alpha}}
\end{gathered}
$$

and where

$$
\begin{gathered}
F_{n}=\frac{1^{\alpha}}{2^{\alpha}} \sum_{m=1}^{\infty} v_{m}\left(v_{m+n}-v_{m-n}\right), \quad n>1, \\
F_{0}=\frac{v_{1}^{2}}{2^{\alpha}} \\
v_{-h}=-v_{h}, \quad h>0 .
\end{gathered}
$$

Because of (39), we can get

$$
\begin{aligned}
& \frac{1^{\alpha}}{2^{\alpha}} \sum_{n=1}^{\infty} u_{n}^{(\alpha)}(t)\left[\cos _{\alpha}\left(n^{\alpha}-1^{\alpha}\right) x^{\alpha}-\cos _{\alpha}\left(n^{\alpha}+1^{\alpha}\right) x^{\alpha}\right] \\
& \quad=\sum_{n=0}^{\infty} E_{n} \cos _{\alpha} n^{\alpha} x^{\alpha}+\frac{1^{\alpha}-\cos _{\alpha} 2 x^{\alpha}}{2^{\alpha}}, \\
& \frac{1^{\alpha}}{2^{\alpha}} \sum_{n=1}^{\infty} v_{n}^{(\alpha)}(t)\left[\cos _{\alpha}\left(n^{\alpha}-1^{\alpha}\right) x^{\alpha}-\cos _{\alpha}\left(n^{\alpha}+1^{\alpha}\right) x^{\alpha}\right] \\
& =\sum_{n=0}^{\infty} F_{n} \cos _{\alpha} n^{\alpha} x^{\alpha}+\frac{1^{\alpha}-\cos _{\alpha} 2 x^{\alpha}}{2^{\alpha}} .
\end{aligned}
$$

Analyzing (37) and equating the coefficient of like $\cos _{\alpha} n^{\alpha} x^{\alpha}(n>1)$ on both sides of equation in (42), respectively, we impose the following assumptions on (42):

$$
u_{n}(t)=v_{n}(t)=0, \quad n>1 \text {. }
$$

Then, equating the coefficient of like $\cos _{\alpha} x^{\alpha}$ on both sides of equation in (42), respectively, the following equation system is obtained:

$$
\begin{gathered}
v_{1}^{(\alpha)}(t)=1^{\alpha}+u_{1} v_{1}, \\
u_{1}^{(\alpha)}(t)=1^{\alpha}+v_{1}^{2} .
\end{gathered}
$$

Analyzing (37), (43), and (44), we can get

$$
u_{1}=v_{1} \text {. }
$$

Considering (44) and (45), we can get

$$
v_{1}^{(\alpha)}(t)=1^{\alpha}+v_{1}^{2} .
$$

Now, we choose the fractional power series method to solve (46).

We assume that the solution $v_{1}(t)$ of (46) can be expressed as a fractional power series in $t^{\alpha}$, as given below:

$$
\begin{aligned}
v_{1}(t) & =\sum_{n=0}^{\infty} v_{1, n} t^{n \alpha} \\
& =v_{1,0}+v_{1,1} t^{\alpha}+v_{1,2} t^{2 \alpha}+\cdots+v_{1, n} t^{n \alpha}+\cdots,
\end{aligned}
$$

where $v_{1, n}, n=0,1,2, \ldots$, are unknown constants to be determined later.

In order to simplify the exposition of the local power series method to solve (46), we first integrate (46) with respect to $t^{\alpha}$ and use the initial condition (37) to get

$$
v_{1}(t)=v_{1}(0)+\int_{0}^{t}\left[1+\left(v_{1}(t)\right)^{2 \alpha}\right](d t)^{\alpha}
$$

Then, substituting (47) into (48), we can easily get

$$
\sum_{n=0}^{\infty} v_{1, n} t^{n \alpha}=v_{1}(0)+\int_{0}^{t}\left[1+\left(\sum_{n=0}^{\infty} v_{1, n} t^{n \alpha}\right)^{2 \alpha}\right](d t)^{\alpha} .
$$

This yields

$$
\begin{aligned}
v_{1,0} & -v_{1}(0)+\left(v_{1,1} t^{\alpha}-t^{\alpha}\right) \\
+ & \sum_{n=2}^{\infty}\left[v_{1, n}-\frac{1^{\alpha}}{n^{\alpha}} \sum_{k=0}^{n-1} v_{1, k} v_{1, n-1-k}\right] t^{n \alpha}=0 .
\end{aligned}
$$

Using the initial condition and equating the coefficients of corresponding powers of $t^{\alpha}$ to zero in (50), we have

$$
v_{1,0}=0, \quad v_{1,1}=1^{\alpha}, \quad v_{1, n}=\frac{1}{n^{\alpha}} \sum_{k=0}^{n-1} v_{1, k} v_{1, n-1-k}
$$

$$
n=2,3, \ldots .
$$


Using the above recursion, the first few components of $v_{1, n}$ are given by

$$
\begin{gathered}
v_{1,0}=0, \\
v_{1,1}=1^{\alpha}, \\
v_{1,2}=0, \\
v_{1,3}=\left(\frac{1}{3}\right)^{\alpha}, \\
v_{1,4}=0, \\
v_{1,5}=\left(\frac{2}{15}\right)^{\alpha}, \\
v_{1,6}=0, \\
v_{1,7}=\left(\frac{17}{315}\right)^{\alpha}
\end{gathered}
$$

According to (5), the final solution $v_{1}(t)$ is thus entirely determined by

$$
\begin{aligned}
v_{1}(t) & =\lim _{n \rightarrow \infty}\left(t^{\alpha}+\frac{t^{3 \alpha}}{3^{\alpha}}+\frac{2^{\alpha} t^{5 \alpha}}{15^{\alpha}}+\frac{17^{\alpha} t^{7 \alpha}}{315^{\alpha}}+\cdots\right) \\
& =\tan _{\alpha} t^{\alpha}
\end{aligned}
$$

Similarly, we can also get

$$
u_{1}(t)=\tan _{\alpha} t^{\alpha}
$$

By virtue of (43), (53), and (54), the final solution of (36) is readily found to be

$$
\begin{aligned}
& u(x, t)=\tan _{\alpha} t^{\alpha} \sin _{\alpha} x^{\alpha}, \\
& v(x, t)=\tan _{\alpha} t^{\alpha} \sin _{\alpha} x^{\alpha} .
\end{aligned}
$$

Example 4. The fractional delay integral-differential equation system is written in the following form:

$$
\begin{aligned}
t^{\alpha} \frac{\partial^{2 \alpha}}{\partial t^{2 \alpha}} u(x, t)= & -4^{\alpha} t^{\alpha} v(x, t) \\
& +16^{\alpha} \int_{0}^{x} u\left(x-\mu, \frac{t}{2}\right) u\left(\mu, \frac{t}{2}\right)(d \mu)^{\alpha}, \\
t^{\alpha} \frac{\partial^{2 \alpha}}{\partial t^{2 \alpha}} v(x, t)=2^{\alpha} u(x, t) & +2^{\alpha} t^{\alpha} \int_{0}^{x} v\left(x-\mu, \frac{t}{2}\right) v\left(\mu, \frac{t}{2}\right)(d \mu)^{\alpha},
\end{aligned}
$$

subject to the boundary and initial conditions described by

$$
\begin{gathered}
\frac{\partial^{\alpha} u}{\partial t^{\alpha}}(x, 0)=\sin _{\alpha} x^{\alpha}, \quad \frac{\partial^{\alpha} v}{\partial t^{\alpha}}(x, 0)=-2^{\alpha} \sin _{\alpha} x^{\alpha}, \\
u(x, 0)=0, \quad v(x, 0)=\sin _{\alpha} x^{\alpha}, \\
u(0, t)=u(\pi, t)=0, \\
v(0, t)=v(\pi, t)=0 .
\end{gathered}
$$

According to (8), (9), and (56), we can get

$$
\begin{aligned}
t^{\alpha} \frac{\partial^{2 \alpha}}{\partial t^{2 \alpha}} u_{n}(t) \sin _{\alpha} n^{\alpha} x^{\alpha}= & -4^{\alpha} t^{\alpha} v_{n}(t) \sin _{\alpha} n^{\alpha} x^{\alpha} \\
& +16^{\alpha} u_{n}^{2}\left(\frac{t}{2}\right) \sin _{\alpha} n^{\alpha} x^{\alpha} \\
t^{\alpha} \frac{\partial^{2 \alpha}}{\partial t^{2 \alpha}} v_{n}(t) \sin _{\alpha} n^{\alpha} x^{\alpha}= & 2^{\alpha} u_{n}(t) \sin _{\alpha} n^{\alpha} x^{\alpha} \\
& +2^{\alpha} t^{\alpha} v_{n}^{2}\left(\frac{t}{2}\right) \sin _{\alpha} n^{\alpha} x^{\alpha} .
\end{aligned}
$$

Equating the coefficient of like $\sin _{\alpha} n^{\alpha} x^{\alpha}$ on both sides of equation in (58), respectively, the following equation system is obtained:

$$
\begin{gathered}
t^{\alpha} \frac{\partial^{2 \alpha}}{\partial t^{2 \alpha}} u_{n}(t)=-4^{\alpha} t^{\alpha} v_{n}(t)+16^{\alpha} u_{n}^{2}\left(\frac{t}{2}\right) \\
t^{\alpha} \frac{\partial^{2 \alpha}}{\partial t^{2 \alpha}} v_{n}(t)=2^{\alpha} u_{n}(t)+2^{\alpha} t^{\alpha} v_{n}^{2}\left(\frac{t}{2}\right)
\end{gathered}
$$

Analyzing (57) and (59), we impose the following assumptions on (59):

$$
u_{n}(t)=v_{n}(t)=0, \quad n=2,3, \ldots
$$

Because of (59), we can get

$$
\begin{gathered}
t^{\alpha} \frac{\partial^{2 \alpha}}{\partial t^{2 \alpha}} u_{1}(t)=-4^{\alpha} t^{\alpha} v_{1}(t)+16^{\alpha} u_{1}^{2}\left(\frac{t}{2}\right), \\
t^{\alpha} \frac{\partial^{2 \alpha}}{\partial t^{2 \alpha}} v_{1}(t)=2^{\alpha} u_{1}(t)+2^{\alpha} t^{\alpha} v_{1}^{2}\left(\frac{t}{2}\right) .
\end{gathered}
$$

Now we choose the fractional power series method to solve (61).

In order to simplify the power series method to solve (61), we first rearrange the two equations in (61) and then integrate them twice with respect to $t^{\alpha}$, respectively. Using the initial condition (57), we can get

$$
\begin{aligned}
u_{1}(t)= & u_{1}(0)+u_{1}^{(\alpha)}(0) t^{\alpha} \\
& +\int_{0}^{t} \int_{0}^{t}\left[-4^{\alpha} v_{1}(t)+\left(\frac{16}{t}\right)^{\alpha} u_{1}^{2}\left(\frac{t}{2}\right)\right](d t)^{\alpha}(d t)^{\alpha}, \\
v_{1}(t)= & v_{1}(0)+v_{1}^{(\alpha)}(0) t^{\alpha} \\
& +\int_{0}^{t} \int_{0}^{t}\left[\frac{2^{\alpha}}{t^{\alpha}} u_{1}(t)+2^{\alpha} v_{1}^{2}\left(\frac{t}{2}\right)\right](d t)^{\alpha}(d t)^{\alpha} ;
\end{aligned}
$$


that is,

$$
\begin{aligned}
u_{1}(t)= & t^{\alpha} \\
& +\int_{0}^{t} \int_{0}^{t}\left[-4^{\alpha} v_{1}(t)+\left(\frac{16}{t}\right)^{\alpha} u_{1}^{2}\left(\frac{t}{2}\right)\right](d t)^{\alpha}(d t)^{\alpha}, \\
v_{1}(t)= & 1^{\alpha}-(2 t)^{\alpha} \\
& +\int_{0}^{t} \int_{0}^{t}\left[\frac{2^{\alpha}}{t^{\alpha}} u_{1}(t)+2^{\alpha} v_{1}^{2}\left(\frac{t}{2}\right)\right](d t)^{\alpha}(d t)^{\alpha} .
\end{aligned}
$$

We assume that the solution $u(t), v(t)$ can be expressed as a fractional power series in $t^{\alpha}$, respectively, as given below:

$$
\begin{aligned}
u_{1}(t)= & \sum_{n=0}^{\infty} u_{1, n} t^{n \alpha}=u_{1,0}+u_{1,1} t^{\alpha} \\
& +u_{1,2} t^{2 \alpha}+\cdots+u_{1, n} t^{n \alpha}+\cdots, \\
v_{1}(t)= & \sum_{n=0}^{\infty} v_{1, n} t^{n \alpha}=v_{1,0} \\
& +v_{1,1} t^{\alpha}+v_{1,2} t^{2 \alpha}+\cdots+v_{1, n} t^{n \alpha}+\cdots,
\end{aligned}
$$

where $u_{1, n}, v_{1, n}, n=0,1,2,3, \ldots$, are unknown constants to be determined later.

Then, substituting (64) into (63), we have

$$
\begin{aligned}
\sum_{n=0}^{\infty} u_{1, n} t^{n \alpha} & \\
=t^{\alpha}+\int_{0}^{t} \int_{0}^{t}[ & -4^{\alpha} \sum_{n=0}^{\infty} v_{1, n} t^{n \alpha} \\
& \left.+\left(\frac{16}{t}\right)^{\alpha}\left(\sum_{n=0}^{\infty} u_{1, n}\left(\frac{t}{2}\right)^{n \alpha}\right)^{2}\right](d t)^{\alpha}(d t)^{\alpha},
\end{aligned}
$$$$
\sum_{n=0}^{\infty} v_{1, n} t^{n}
$$$$
=1^{\alpha}-(2 t)^{\alpha}
$$$$
+\int_{0}^{t} \int_{0}^{t}\left[\frac{2^{\alpha}}{t^{\alpha}} \sum_{n=0}^{\infty} u_{1, n} t^{n \alpha}\right.
$$$$
\left.+2^{\alpha}\left(\sum_{n=0}^{\infty} v_{1, n}\left(\frac{t}{2}\right)^{n \alpha}\right)^{2}\right](d t)^{\alpha}(d t)^{\alpha} .
$$

This yields

$$
\begin{aligned}
u_{1,0}+u_{1,1} t^{\alpha}-t^{\alpha}+\sum_{n=2}^{\infty} u_{1, n} t^{n \alpha} \\
=\int_{0}^{t} \int_{0}^{t}\left[-4^{\alpha} \sum_{n=0}^{\infty} v_{1, n} t^{n \alpha}\right. \\
\left.+\left(\frac{16}{t}\right)^{\alpha}\left(\sum_{n=0}^{\infty} u_{1, n}\left(\frac{t}{2}\right)^{n \alpha}\right)^{2}\right](d t)^{\alpha}(d t)^{\alpha},
\end{aligned}
$$

$$
\begin{aligned}
v_{1,0}+v_{1,1} t^{\alpha}-1^{\alpha}+(2 t)^{\alpha}+\sum_{n=2}^{\infty} v_{1, n} t^{n \alpha} \\
=\int_{0}^{t} \int_{0}^{t}\left[\left(\frac{2}{t}\right)^{\alpha} \sum_{n=0}^{\infty} u_{1, n} t^{n \alpha}\right. \\
\left.+2^{\alpha}\left(\sum_{n=0}^{\infty} v_{1, n}\left(\frac{t}{2}\right)^{n \alpha}\right)^{2}\right](d t)^{\alpha}(d t)^{\alpha} .
\end{aligned}
$$

According to (66), we can get

$$
\begin{gathered}
u_{1,0}+u_{1,1} t^{\alpha}-t^{\alpha} \\
+\sum_{n=2}^{\infty}\left(u_{1, n}+4^{\alpha} \frac{v_{1, n-2}}{n^{\alpha}(n-1)^{\alpha}}\right. \\
\left.\quad-\frac{16^{\alpha}}{2^{(n-1) \alpha} n^{\alpha}(n-1)^{\alpha}} \sum_{k=0}^{n-1} u_{1, k} u_{1, n-k-1}\right) t^{n \alpha}=0, \\
v_{1,0}+v_{1,1} t^{\alpha}-1^{\alpha}+(2 t)^{\alpha} \\
+\sum_{n=2}^{\infty}\left(v_{1, n}-2^{\alpha} \frac{u_{1, n-1}}{n^{\alpha}(n-1)^{\alpha}}\right. \\
\left.-\frac{2^{\alpha}}{2^{(n-2) \alpha} n^{\alpha}(n-1)^{\alpha}} \sum_{k=0}^{n-2} v_{1, k} v_{1, n-k-2}\right) t^{n \alpha}=0 .
\end{gathered}
$$

Equating the coefficients of corresponding powers of $t^{\alpha}$ to zero in (67), we have

$$
\begin{aligned}
& u_{1, n}=-4^{\alpha} \frac{v_{1, n-2}}{n^{\alpha}(n-1)^{\alpha}} \\
&+\frac{16^{\alpha}}{2^{(n-1) \alpha} n^{\alpha}(n-1)^{\alpha}} \sum_{k=0}^{n-1} u_{1, k} u_{1, n-k-1}, \\
& v_{1, n}= 2^{\alpha} \frac{u_{1, n-1}}{n^{\alpha}(n-1)^{\alpha}} \\
&+\frac{2^{\alpha}}{2^{(n-2) \alpha} n^{\alpha}(n-1)^{\alpha}} \sum_{k=0}^{n-2} v_{1, k} v_{1, n-k-2}, \\
& u_{1,0}=0, \quad u_{1,1}=1^{\alpha}, \\
& v_{1,0}=1^{\alpha}, \quad v_{1,1}=-2^{\alpha} .
\end{aligned}
$$

From above recursion, first few components of $u_{1, n}, v_{1, n}$ are given by

$$
\begin{gathered}
u_{1,0}=0, \quad u_{1,1}=1^{\alpha}, \quad u_{1,2}=-2^{\alpha}, \\
u_{1,3}=2^{\alpha}, \quad u_{1,4}=-\left(\frac{4}{3}\right)^{\alpha}, \quad u_{1,5}=\left(\frac{2}{3}\right)^{\alpha}, \\
u_{1,6}=-\left(\frac{4}{15}\right)^{\alpha}, \ldots ; \\
v_{1,0}=1^{\alpha}, \quad v_{1,1}=-2^{\alpha}, \quad v_{1,2}=2^{\alpha},
\end{gathered}
$$




$$
\begin{gathered}
v_{1,3}=-\left(\frac{4}{3}\right)^{\alpha}, \quad v_{1,4}=\left(\frac{2}{3}\right)^{\alpha}, \quad v_{1,5}=-\left(\frac{4}{15}\right)^{\alpha}, \\
v_{1,6}=\left(\frac{4}{45}\right)^{\alpha}, \ldots
\end{gathered}
$$

According to (5), the final solution $u_{1}(t), v_{1}(t)$ is thus entirely determined by

$$
\begin{aligned}
& u_{1}(t) \\
& =\lim _{n \rightarrow \infty}\left(t^{\alpha}-2^{\alpha} t^{2 \alpha}+2^{\alpha} t^{3 \alpha}-\left(\frac{4}{3}\right)^{\alpha} t^{4 \alpha}+\left(\frac{2}{3}\right)^{\alpha} t^{5 \alpha}-\cdots\right) \\
& =t^{\alpha} E_{\alpha}(-2 t)^{\alpha}, \\
& v_{1}(t) \\
& =\lim _{n \rightarrow \infty}\left(1^{\alpha}-2^{\alpha} t^{\alpha}+2^{\alpha} t^{2 \alpha}-\left(\frac{4}{3}\right)^{\alpha} t^{3 \alpha}+\left(\frac{2}{3}\right)^{\alpha} t^{4 \alpha}\right. \\
& \left.\quad-\left(\frac{4}{15}\right)^{\alpha} t^{5 \alpha}+\left(\frac{4}{45}\right)^{\alpha} t^{6 \alpha} \ldots\right) \\
& =E_{\alpha}(-2 t)^{\alpha} .
\end{aligned}
$$

By virtue of (60) and (70), the final solution of (56) is readily found to be

$$
\begin{gathered}
u(x, t)=t^{\alpha} E_{\alpha}(-2 t)^{\alpha} \sin _{\alpha} x^{\alpha}, \\
v(x, t)=E_{\alpha}(-2 t)^{\alpha} \sin _{\alpha} x^{\alpha} .
\end{gathered}
$$

\section{Conclusion}

In this paper, we have presented and implemented the local fractional Fourier series method to solve the nonlinear equations problems. It is a straightforward and convenient algorithm for deriving particular solution of some nonlinear fractional integral-differential or differential equations with the boundary problem. However, as other methods, this method has its own deficiency; for example, there is some difficulty in calculating the local fractional Fourier series of the nonlinear part of the equation, which may narrow down its applications.

\section{Conflict of Interests}

The authors declare that they have no conflict of interests regarding this paper.

\section{Acknowledgments}

This work is supported by the Foundation and Advanced Technology Research Program of Henan province (142300410385, 142300410384, 132300410211, and 132300410440) and the Nanyang Normal University (NYNU2005k37). The authors would like to thank the editor and the reviewers for their constructive comments and suggestions to improve the quality of the paper.

\section{References}

[1] S. G. Samko, A. A. Kilbas, and O. I. Marichev, Integrals and Derivatives of Fractional Order and Applications, Nauka i Tehnika, Minsk, Belarus, 1987.

[2] A. A. Kilbas, H. M. Srivastava, and J. J. Trujillo, Theory and Applications of Fractional Differential Equations, Elsevier, Amsterdam, The Netherlands, 2006.

[3] D. Baleanu, A. K. Golmankhaneh, and A. K. Golmankhaneh, "Solving of the fractional non-linear and linear Schroedinger equations by homotopy perturbation method," Romanian Journal of Physics, vol. 54, no. 9-10, pp. 823-832, 2009.

[4] H. Jafari, K. Sayevand, H. Tajadodi, and D. Baleanu, "Homotopy analysis method for solving Abel differential equation of fractional order," Central European Journal of Physics, vol. 11, no. 10, pp. 1523-1527, 2013.

[5] B. Jang, "Efficient analytic method for solving nonlinear fractional differential equations," Applied Mathematical Modelling, vol. 38, no. 5-6, pp. 1775-1787, 2014.

[6] A. Elsaid, "Fractional differential transform method combined with the Adomian polynomials," Applied Mathematics and Computation, vol. 218, no. 12, pp. 6899-6911, 2012.

[7] H. Jafari and V. Daftardar-Gejji, "Positive solutions of nonlinear fractional boundary value problems using Adomian decomposition method," Applied Mathematics and Computation, vol. 180, no. 2, pp. 700-706, 2006.

[8] A. H. Bhrawy and M. M. Al-Shomrani, "A shifted Legendre spectral method for fractional-order multi-point boundary value problems," Advances in Difference Equations, vol. 2012, article 8, 2012.

[9] G. C. Wu and D. Baleanu, "New applications of the variational iteration method-from differential equations to $q$-fractional difference equations," Advances in Difference Equations, vol. 2013, article 21, 16 pages, 2013.

[10] G.-C. Wu and D. Baleanu, "Variational iteration method for the Burgers' flow with fractional derivatives-new Lagrange multipliers," Applied Mathematical Modelling, vol. 37, no. 9, pp. 6183-6190, 2013.

[11] G.-C. Wu, "A fractional variational iteration method for solving fractional nonlinear differential equations," Computers \& Mathematics with Applications, vol. 61, no. 8, pp. 2186-2190, 2011.

[12] A. H. Bhrawy and M. A. Alghamdi, "A shifted Jacobi-GaussLobatto collocation method for solving nonlinear fractional Langevin equation involving two fractional orders in different intervals," Boundary Value Problems, vol. 2012, article 62, 2012.

[13] X. J. Yang, Local Fractional Functional Analysis and Its Applications, Asian Academic Publisher, Hong Kong, 2011.

[14] X. J. Yang, Advanced Local Fractional Calculus and Its Applications, World Science Publisher, New York, NY, USA, 2012.

[15] J. H. He, S. K. Elagan, and Z. B. Li, "Geometrical explanation of the fractional complex transform and derivative chain rule for fractional calculus," Physics Letters A, vol. 376, no. 4, pp. 257259, 2012.

[16] J.-H. He, "A new fractal derivation," Thermal Science, vol. 15, supplement 1, pp. S145-S147, 2011.

[17] Y.-Y. Li, Y. Zhao, G.-N. Xie, D. Baleanu, X.-J. Yang, and K. Zhao, "Local fractional Poisson and Laplace equations with applications to electrostatics in fractal domain," Advances in Mathematical Physics, vol. 2014, Article ID 590574, 5 pages, 2014. 
[18] Y. Zhao, D. Baleanu, C. Cattani, D.-F. Cheng, and X.-J. Yang, "Maxwell's equations on Cantor sets: a local fractional approach," Advances in High Energy Physics, vol. 2013, Article ID 686371, 6 pages, 2013.

[19] X.-J. Yang, D. Baleanu, Y. Khan, and S. T. Mohyud-Din, "Local fractional variational iteration method for diffusion and wave equations on Cantor sets," Romanian Journal of Physics, vol. 59, no. 1-2, pp. 36-48, 2014.

[20] Y. Zhang, A. Yang, and X.-J. Yang, "1-D heat conduction in a fractal medium: a solution by the local fractional Fourier series method," Thermal Science, vol. 17, no. 3, pp. 953-956, 2013.

[21] M.-S. Hu, R. P. Agarwal, and X.-J. Yang, "Local fractional Fourier series with application to wave equation in fractal vibrating string," Abstract and Applied Analysis, vol. 2012, Article ID 567401, 15 pages, 2012.

[22] S.-Q. Wang, Y.-J. Yang, and H. K. Jassim, "Local fractional function decomposition method for solving inhomogeneous wave equations with local fractional derivative," Abstract and Applied Analysis, vol. 2014, Article ID 176395, 7 pages, 2014.

[23] H. M. Srivastava, A. K. Golmankhaneh, D. Baleanu, and X.-J. Yang, "Local fractional Sumudu transform with application to IVPs on Cantor sets," Abstract and Applied Analysis, vol. 2014, Article ID 620529, 7 pages, 2014.

[24] X.-J. Yang, J. Hristov, H. M. Srivastava, and B. Ahmad, "Modelling fractal waves on shallow water surfaces via local fractional Korteweg-de Vries equation," Abstract and Applied Analysis, vol. 2014, Article ID 278672, 10 pages, 2014. 


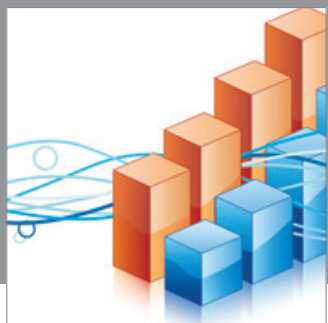

Advances in

Operations Research

mansans

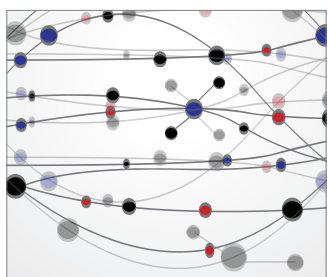

The Scientific World Journal
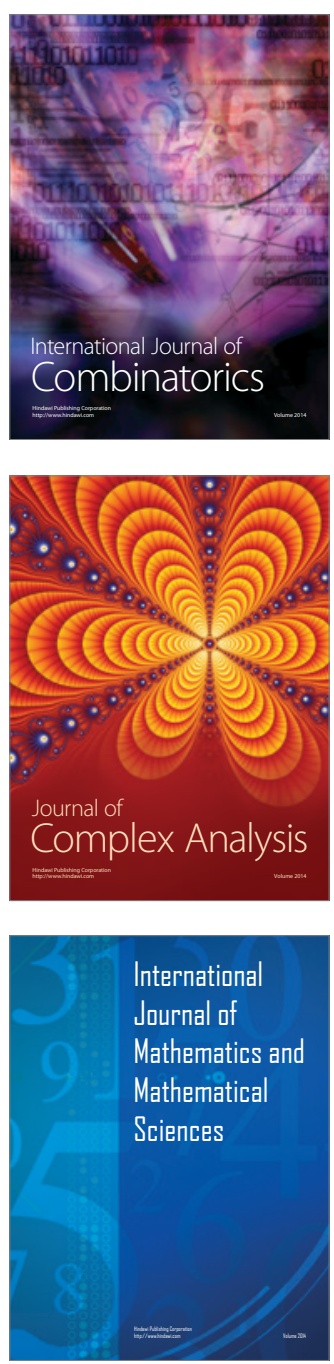
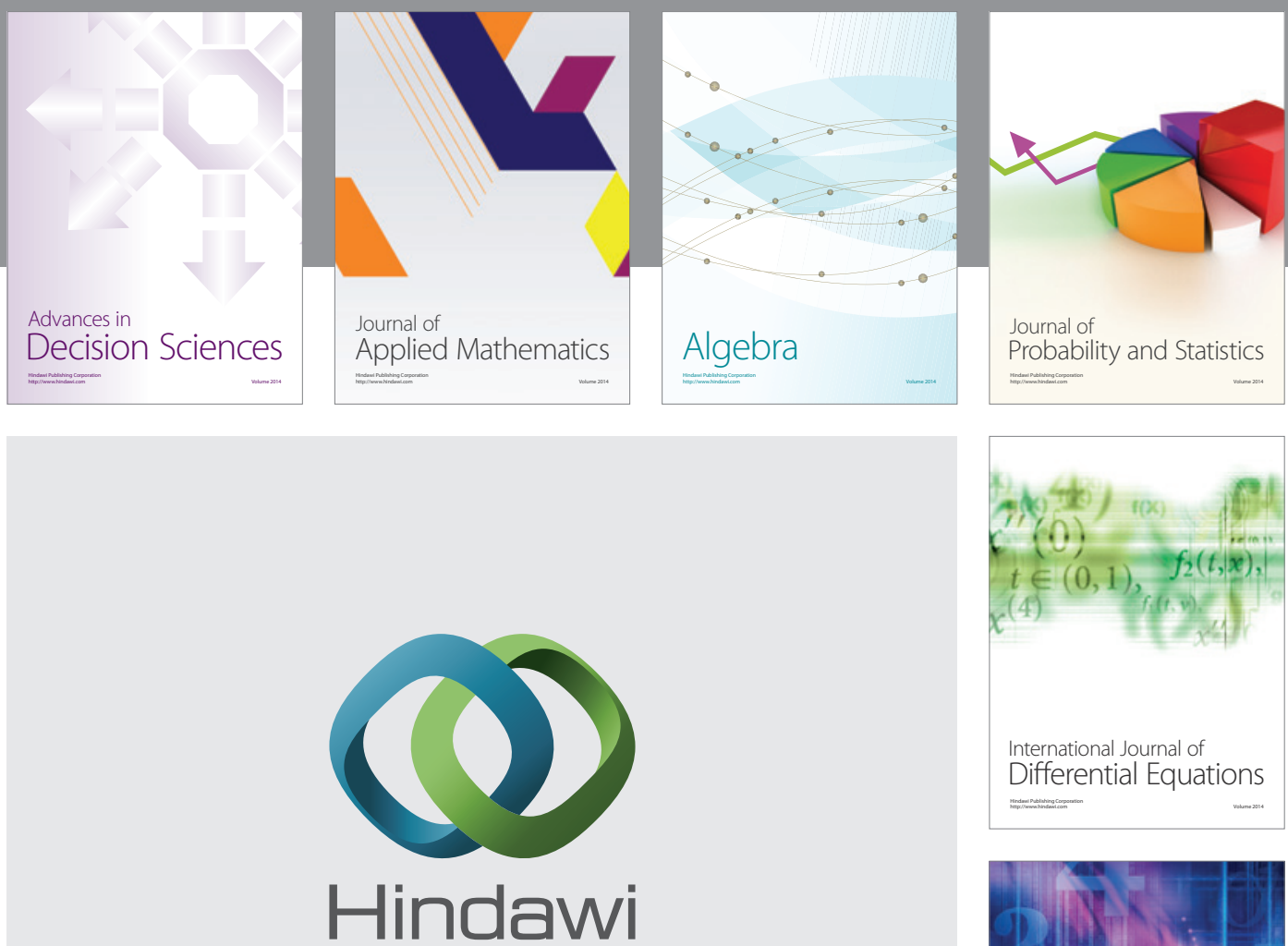

Submit your manuscripts at http://www.hindawi.com
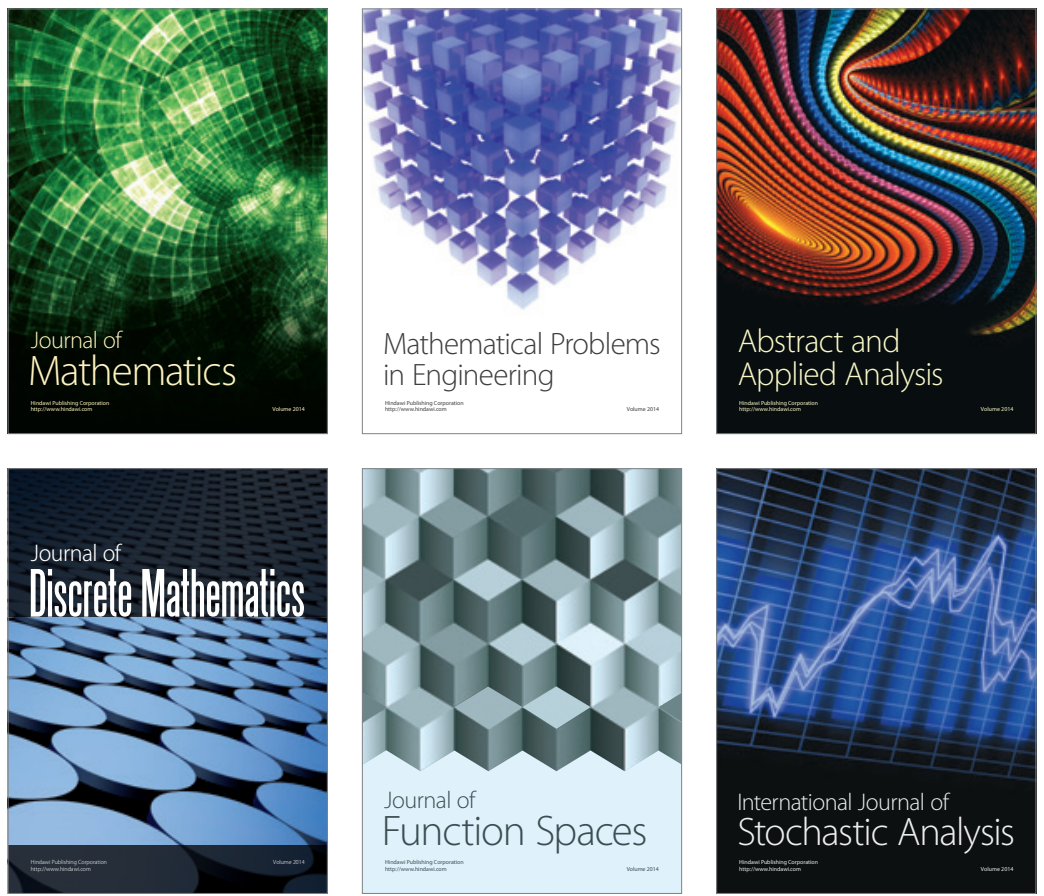

Journal of

Function Spaces

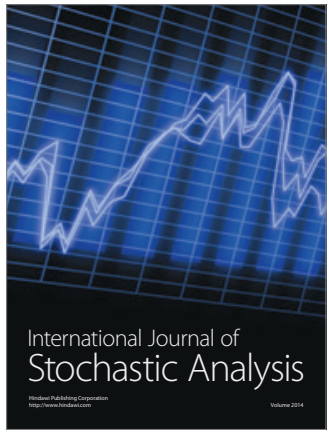

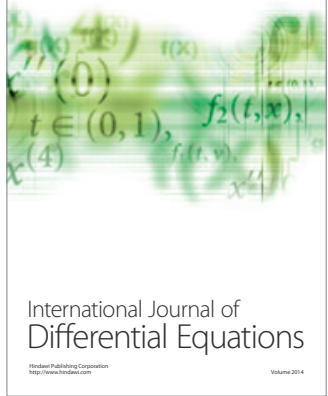
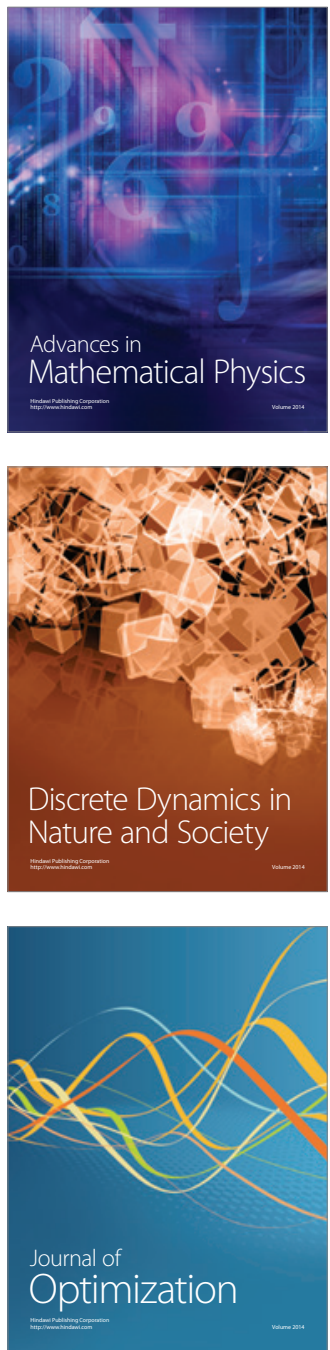